\title{
Optimal Combination of Transmission Parameters for Maximizing the Throughput of WLAN
}

\author{
Pravinkumar Patil, Meenakshi Patil, Santosh Itraj, Uttam Bombale
}

\begin{abstract}
In wireless local area networks (WLANs), the data transmission rate is highly wavering concerning time according to changes in the channel environment. The traditional link adaptation algorithms, which rely on channel state information (CSI) estimation for selecting an appropriate modulation-coding scheme (MCS) does not thrive with madly varying channel conditions. To uphold this problem, in this paper we contemplate the link adaptation problem in IEEE 802.11n based WLAN by evaluating the performance under different combinations of transmission parameters or transmit modes. The evaluation results show that an optimal selection of transmission mode on a per-packet basis according to the current channel state maximizes the throughput performance of WLAN. Simulations over a wide class of TGn fading channel model shows that significant improvement in the throughput is possible by selecting an optimal combination of transmission parameters adaptively on a per-packet basis according to SNR estimation made at receiver.
\end{abstract}

Keywords: BER, IEEE802.11n, MIMO, OFDM, Throughput, TGn channel model, WLAN.

\section{INTRODUCTION}

Wireless communication suffers greatly from vast instability in the radio environment between the access points (AP) and communicating node. Packet loss or bit error rate (BER) resulting from the multipath fading effects degrade the performance of WLANs. To enable reliable transmission under the worst conditions in the radio path, the link adaptive transmission method in WLAN switches to the most robust MCS. When the estimated channel condition in terms of signal to noise ratio (SNR) is better, the rate adaptation scheme selects the MCS that yield higher data rate under the constraint of quality of service (QoS) requirements. The link adaptation methods for WLAN presented in [1] [2] are based on adaptive modulation-coding (AMC) based rate selection. The link adaptation problem in WLAN is an extensively studied subject in the research literature. IEEE 802.11n is the prominent WLAN standard in use today. The new physical

Revised Manuscript Received on February 15, 2020.

* Correspondence Author

Pravinkumar Patil*, VTU, Belagavi, India. E-mail: pspatil108@gmail.comDr. Meenakshi Patil, VTU, Belagavi, India. E-mail: meenakshirpatil@gmail.com

Santosh Itraj, D.O.T., Shivaji University, Kolhapur, India. E-mail: ssitraj@yahoo.com

Dr. Uttam Bombale, D.O.T., Shivaji University, Kolhapur, India. E-mail: uttam bombale@rediffmail.com

(C) The Authors. Published by Blue Eyes Intelligence Engineering and Sciences Publication (BEIESP). This is an open access article under the CC BY-NC-ND license (http://creativecommons.org/licenses/by-nc-nd/4.0/) layer enhancements such as multiple-input multiple-output (MIMO), channel bonding, extended MCS modes, and improved orthogonal frequency

division multiplexing (OFDM) are included in the IEEE 802.11n amendment [3]. The link adaptation proposals for legacy WLAN standards such as IEEE $802.11 \mathrm{a} / \mathrm{b} / \mathrm{g}$ work out to be less effective for $802.11 \mathrm{n}$. The recent link adaptation proposal ARAMIS [4] which jointly adapts data rate and channel bandwidth, has significantly increased throughput. The researchers in [5] introduced a new link adaptation scheme that zigzags between two modes (inter-mode and intra-mode) for selecting the most appropriate rate. Authors in [6] investigated the effect of link adaptation based on MIMO mode, frame length, and channel bandwidth on the performance of 802.11n. The adaptive proposal for 802.11n based WLAN called MiSer [7] uses a rate-power pair's lookup table for link appropriate switching between rate-power combinations for maximizing throughput. The interference aware adaptation algorithm introduced in [8] jointly adapts rate and channel. All these link adaptation schemes are based on the estimation of CSI or received signal strength indicator (RSSI). In this paper the throughput and BER performance of IEEE 802.11n based WLAN is tested under different combination of physical parameters. The simulations are carried out over wide class of TGn channel models considering smaller bins of applicable SNR range. The objective of this paper is to determine an optimal combination of transmission parameters for different SNR bins.The rest of the paper is organized as follows. Section II explains the physical layer enhancements of IEEE $802.11 \mathrm{n}$ WLAN. Section III discuss the TGn channel model. AMC based rate adaptation method using estimated signal to noise ratio ( $\mathrm{SNR}_{\mathrm{est}}$ ) is discussed in section IV. The discussion on the different transmission modes that we have considered in this paper is included in section V. Section VI presents simulation results carried over a wide variety of TGn fading channels for different transmission modes and the concluding remarks are given in section VII.

\section{WLAN PHYSICAL LAYER}

The IEEE $802.11 \mathrm{n}$ is today's prominent WLAN standard. The details of WLAN 802.11n specifications can be found in [3]. The Physical layer specifications of this WLAN standard are based on MIMO OFDM technology [9] [10]. OFDM changes frequency selective fading channel scenario into a frequency non-selective also called flat fading one and helps in mitigating wideband multipath fading effects [11][12]. The OFDM implementation is based on modulation using Inverse Fast Fourier transform

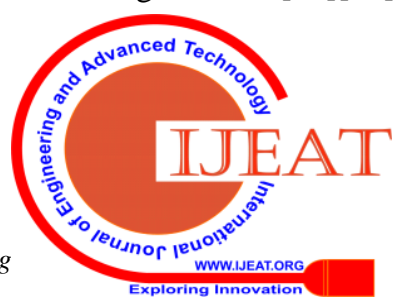


(IFFT) at the transmitting end and demodulation through Fast Fourier Transform (FFT) at the receiver. In OFDM symbol 52 of total 64 sub-carriers carry data and 4 are pilot sub-carriers. The sub-carrier frequency separation is 3.125 $\mathrm{KHz}$ and the wideband channel width is $20 \mathrm{MHz}$. To ensure orthogonality between sub-carriers and to eliminate Inter Symbol Interference (ISI) end portion of the OFDM symbol is prefixed to act as guard interval. IEEE 802.11n amendment [3] [13] defines two guard intervals viz. Long (equal to $800 \mathrm{~ns}$ ) and Short (equal to 400ns). The MIMO technology in 802.11n supports using space-time block coding (STBC) and/or spatial division multiplexing (SDM) based diversity coding using a maximum of 4 spatial streams. The MCS based transmission modes use a combination of different modulation (BPSK, QPSK, QAM-16, or QAM-64) and binary convolutional coding (BCC) with code rate $(1 / 2,2 / 3$, $3 / 4$, or $5 / 6$ ) [12][11]. The details of MCS based transmission modes and corresponding maximum capacity in Mbps is given in [3]. Existing WLAN chipsets are using AMC based link adaptation method to choose MCS most appropriate to present the link state. Fig. 1 depicts the high throughput mixed with legacy (HT-mixed) packet structure [14].where L-STF is legacy short training field, L-LTF is legacy long training field, and L-SIG is legacy signal field. Table I presents details of the HT-PPDU field functions.

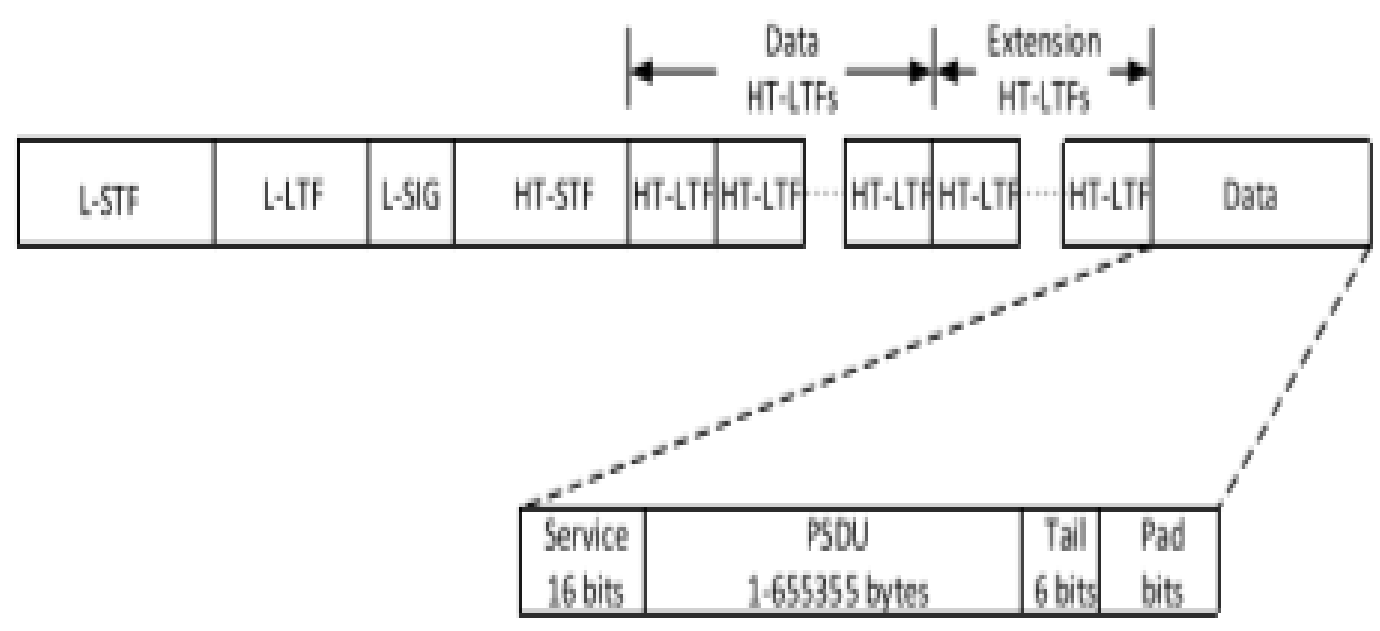

Fig 1. HT-mixed packet structure

Table- I: Non legacy HT-PPDU field functions

\begin{tabular}{|c|c|c|c|}
\hline Field Name & Description & Function & $\begin{array}{c}\text { Time in } \\
\text { Microseconds }\end{array}$ \\
\hline HT-SIG & HT signal field & $\begin{array}{c}\text { Carries information required for decoding HT } \\
\text { length, FEC encoding type, guard interval, } \\
\text { number of extension spatial streams, etc. Used for } \\
\text { auto detection between legacy OFDM packets and } \\
\text { HT-mixed frame format. }\end{array}$ & $\begin{array}{c}\text { To improve AGC estimation for MIMO system. } \\
\text { HT-STF }\end{array}$ \\
\hline HT-LTF & HT short training field & To estimate MiMO channel & 4 \\
\hline HT-Data & HT long training field & HT data field & $\begin{array}{c}\text { Carries MAC layer frames. Comprises of service } \\
\text { field, PSDU, tail bits, and pad bits. }\end{array}$ \\
\hline
\end{tabular}

\section{CHANNEL MODEL}

TGn channel model is a set of MIMO broadband channel models developed by Task Group n (TGn) for IEEE 802.11n. The series of measurements taken both, in indoor and outdoor radio environments are used to describe the characteristic of TGn channel models. There is six variant of this channel model differing in the length of impulse response and the number of clusters that are considered. Each channel model profile in this set represents a specific radio channel scenario. These six different TGn channel profiles are denoted with alphabets A through F. The most commonly used TGn channel profiles are B, C, D, and E. Table II summarizes the characteristic of different TGn channel models [15]. 
Table- II: Characteristic of different TGn channel models

\begin{tabular}{|c|c|c|c|}
\hline $\begin{array}{c}\text { Channel } \\
\text { model }\end{array}$ & Environment & Condition & $\begin{array}{c}\text { RMS delay } \\
\text { spread (ns) }\end{array}$ \\
\hline A & LOS/NLOS & Residential & 0 \\
\hline B & LOS/NLOS & Residential & 15 \\
\hline C & LOS/NLOS & $\begin{array}{c}\text { Residential/ Small } \\
\text { office }\end{array}$ & 30 \\
\hline D & LOS/NLOS & $\begin{array}{c}\text { Small or typical } \\
\text { office / Large } \\
\text { office }\end{array}$ & 50 \\
\hline E & NLOS & Large office & 100 \\
\hline F & LOS/NLOS & $\begin{array}{c}\text { Large office/ } \\
\text { Large space }\end{array}$ & 150 \\
\hline
\end{tabular}

\section{RATE ADAPTATION}

IEEE 802.11n WLAN standard supports multiple mcs as given in the table IV. The adaptation of mcs in WLAN according to channel variations results in a notable capacity gain. The rate adaptation (RA) mechanism becomes now an integrated process in the IEEE 802.11n based chipsets. Adaptive modulation-coding (AMC) based rate adaptation in WLAN is a highly researched subject in the research literature. The existing RA algorithms are classified as open loop and closed loop. Most of the closed-loop RA algorithms are receiver-based and depend on estimations of CSI, SNR, or RSSI for appropriate mcs selection. The SNR based RA algorithms use the thresholding method as shown in the table III to switch between different mcs. The SNR switching thresholds can be determined using either theoretical or empirical methods. The performance of the RA algorithm largely depends on the accuracy of the SNR estimation method. We use different SNR switching thresholds for different transmission modes to select more appropriate mcs to maximize throughput in a vast varying channel environment.

Table- III: Lookup table for SNR based rate adaption

\begin{tabular}{|c|c|}
\hline mcs index & snr $_{\text {est }}$ \\
\hline 0 & $0<\mathrm{snr}_{\text {est }}<=\mathrm{T} 1$ \\
\hline 1 & $\mathrm{~T} 1<\mathrm{snr}_{\text {est }}<=\mathrm{T} 2$ \\
\hline 2 & $\mathrm{~T} 2<\mathrm{snr}_{\text {est }}<=\mathrm{T} 3$ \\
\hline 3 & $\mathrm{~T} 3<\mathrm{snr}_{\text {est }}<=\mathrm{T} 4$ \\
\hline 4 & $\mathrm{~T} 4<\mathrm{snr}_{\text {est }}<=\mathrm{T} 5$ \\
\hline 5 & $\mathrm{~T} 5<\mathrm{snr}_{\text {est }}<=\mathrm{T} 6$ \\
\hline 6 & $\mathrm{~T} 6<\mathrm{snr}_{\text {est }}<=\mathrm{T} 7$ \\
\hline 7 & snr $_{\text {est }}>\mathrm{T} 7$ \\
\hline
\end{tabular}

\section{TRANSMISSION MODES}

The WLAN performance is analysed considering the following transmission modes.

1. RA-SISO: Rate adaptive single input single output

2. RA-STBC: Rate adaptive space-time block coding

3. RA-SDM: Rate adaptive spatial division multiplexing

The table IV presents different combinations of transmission parameters and corresponding capacity of the WLAN system.

Table- IV: Transmission parameters and capacity of IEEE 802.11n WLAN system

\begin{tabular}{|l|c|c|c|c|c|}
\hline Modulation & Code & \multicolumn{4}{|c|}{ Data Rate capacity in Mbps } \\
\cline { 3 - 6 } & Rate & \multicolumn{2}{|c|}{ SISO or STBC } & \multicolumn{2}{|c|}{ SDM } \\
\cline { 3 - 6 } & & SG $=$ & LG & SG $=400 \mathrm{~ns}$ & LG $=$ \\
& & $400 \mathrm{~ns}$ & $800 \mathrm{~ns}$ & & $800 \mathrm{~ns}$ \\
\hline
\end{tabular}

\begin{tabular}{|c|c|c|c|c|c|}
\hline bpsk & $1 / 2$ & 7.2 & 6.5 & 14.4 & 13 \\
\hline qpsk & $1 / 2$ & 14.4 & 13 & 28.8 & 26 \\
\hline qpsk & $3 / 4$ & 21.7 & 19.5 & 43.4 & 39 \\
\hline $16-q a m$ & $1 / 2$ & 28.9 & 26 & 57.8 & 52 \\
\hline $16-q a m$ & $3 / 4$ & 43.3 & 39 & 86.6 & 78 \\
\hline 64-qam & $2 / 3$ & 57.8 & 52 & 115.6 & 104 \\
\hline 64-qam & $3 / 4$ & 65 & 58.5 & 130 & 117 \\
\hline 64-qam & $5 / 6$ & 72.2 & 65 & 144.4 & 130 \\
\hline
\end{tabular}

\section{A. RA-SISO}

RA-SISO with either long or short guard interval is the better transmission mode over the middle SNR range. This mode uses single transmit and single receive antenna for transmission. In this mode of transmission link, appropriate mcs is selected adaptively based on estimate SNR at the receiving end.

\section{Effect on the BER}

The BER of the WLAN system is the function of the selected modulation scheme as well as SNR. The BER performance of the WLAN with RA-SISO transmission mode is the function of the present state of radio channel $\left(\mathrm{SNR}_{\mathrm{est}}\right)$ and the selected modulation scheme. The BER equations as a function of modulation scheme and the signal to noise ratio per bit (EbNo) for Rayleigh fading channel presented by Simon in [16] are modified as given below.

\section{BER for bpsk modulation}

$$
\left.P_{e}\left(s n r_{e s t}, m c s\right)\right|_{b p s k}=\frac{1}{2}\left[1-\sqrt{\frac{\left(\frac{B W}{\text { BitRate }}\right) s n r_{\text {est }}}{1+\left(\frac{B W}{\text { BitRate }}\right) s n r_{\text {est }}}}\right]
$$

Where $S N R_{e s t}$ is average estimation of the received signal to noise ratio; $B W$ is the bandwidth of the channel; and BitRate is the maximum possible capacity of WLAN system with selected mcs.

\section{BER for M-qam modulation}

$$
\left.P_{e}\left(s n r_{\text {est }}, m c s\right)\right|_{M-q a m}=\left(2\left(1-\frac{1}{\sqrt{M}}\right) \frac{1}{\log _{2}}\right) \cdot \beta
$$

Where,

$$
\beta=\sum_{i=1}^{\sqrt{\frac{M}{2}}}\left(1-\sqrt{\frac{1.5(2 i-1)^{2 \backslash}\left(\frac{B W}{\text { BitRate }}\right) \cdot s n r_{\text {est }} \cdot \log _{2} M}{M-1+1.5(2 i-1)^{2 \backslash}\left(\frac{B W}{\text { BitRate }}\right) \cdot \text { snr }_{\text {est }} \cdot \log _{2} M}}\right)
$$

Equations (1) to (3) are used to find SNR thresholds vector for targeted BER of $10^{-1}$ as given below.

$$
T=\left[\begin{array}{llllllll}
T_{1} & T_{2} & T_{3} & T_{4} & T_{5} & T_{6} & T_{7} & T_{8}
\end{array}\right]
$$




\section{Effect on the throughput}

The throughput of the WLAN system depends on the BER as given in the following equation.

$$
\text { Throughput }=\text { BitRate } \cdot\left(1-P_{e}\right)
$$

\section{B. RA-STBC}

The space time-block coding (STBC) makes the system more robust against bit errors resulted due to the fading radio environment. In the lower SNR range, where RA-SDM is not satisfying the BER requirement (less than 0.1 in our case), the RA-STBC with either short or long guard interval is the only better transmission mode. Therefore, irrespective of the throughput that we get using other transmission modes, the RA-STBC is considered as an optimal mode for transmission in the lower SNR range. This mode of transmission uses rate adaptation with Alamouti coding [17]

\section{Improvement in the BER performance}

RA-STBC mode improves the BER performance of the system. The improvement in the BER performance resulting due to STBC encoding is called diversity gain as given in the following equation.

$$
P_{e_{(\text {STBC })}}=\left(l_{l}^{2 l-1}\right)\left(P_{e}\left(s n r_{e s t}, m c s\right)\right)^{l}
$$

Where $l$ is called diversity order which is defined as follows. In $2 \mathrm{X} 1 \mathrm{STBC}$ encoding as $N t=2, N r=1$, and $l$ will take value equal to 2 . The BER equation in (6) therefore is modified as given below.

$$
P_{e_{(S T B C)}}=3\left(P_{e}\left(s n r_{\text {est }}, m c s\right)\right)^{2}
$$

\section{RA-SDM}

The spatial division multiplexing (SDM) encoding for MIMO improves significantly the throughput performance of WLAN. However, the channel condition must be good enough to consider this mode of operation for transmission. Under high SNR conditions, this mode allows parallel transmission over multiple spatial streams. The SDM encoding method is well explained in [10] [18].

\section{Improvement in the throughput performance}

The measure of the throughput gain as a result of SDM encoding is called multiplexing gain, which is defined in the following equation.

$$
\text { Throughput }_{S D M}=G_{m} \cdot\left(\text { Throughput }_{\text {SISO }}\right)
$$

Where $G_{m}$ is the gain due to multiplexing which is equal to the number of spatial streams. In 2X2 MIMO based SDM encoding the number of spatial streams is 2 and therefore the throughput gets doubled as given in the following equation.

$$
\text { Throughput }_{S D M(2 X 2)}=2 \cdot\left(\text { Throughput }_{\text {SISO }}\right)
$$

\section{SIMULATION RESULTS AND DISCUSSIONS}

Fig. 3 to Fig. 6 on show simulations carried out over variants of TGn channel models (TGn B, TGn C, and TGn D) with a different combination of transmission parameters. The average throughput performance of WLAN with different transmission modes calculated over smaller SNR bins are presented in tables V to VII.

\section{A. Performance of RA-SISO}

Although RA-SDM is an optimal transmission mode in terms of throughput, it requires good channel conditions. When the SNR is less than $30 \mathrm{~dB}$, RA-SDM mode results in increased interference which reduces the ultimate throughput of the system. As shown in the Fig. 2, Fig. 4, and Fig. 6 in all channel scenarios RA-SISO with long or short guard interval is found to be optimal in terms of throughput as well as BER when the SNR varies between $15 \mathrm{~dB}$ to $30 \mathrm{~dB}$. Tables $\mathrm{V}$ to VII shows that the performance of WLAN with RA-SISO transmission mode is better than other transmission modes over the middle SNR range.

\section{B. Performance of RA-STBC}

When it is a small or typical office environment, the TGn C or TGn D is the appropriate channel model to consider [15]. In these channel scenario, RA-STBC with long guard interval is the only transmission mode which satisfies the BER constraint (BER $<0.1$ in our case) when SNR is less than 15 $\mathrm{dB}$ as shown in Fig. 5 and Fig. 7. Therefore irrespective of throughput that resulted due to other transmission mode, RA-STBC is selected as an optimal mode for transmission as presented in table VI and VII.

\section{Performance of RA-SDM}

For TGn-D channel model (appropriate for large office environment with NLOS communication), RA-SDM with short guard interval is found to be an optimal transmission mode in lower (SNR $<15 \mathrm{~dB}$ ) as well as higher (SNR $>30$ $\mathrm{dB})$ SNR range in terms of average throughput result as shown in Fig 6. In all other channel scenarios, RA-SDM outperforms other modes when the channel condition is good enough (SNR > $30 \mathrm{~dB}$ ) as presented in table V to VII.

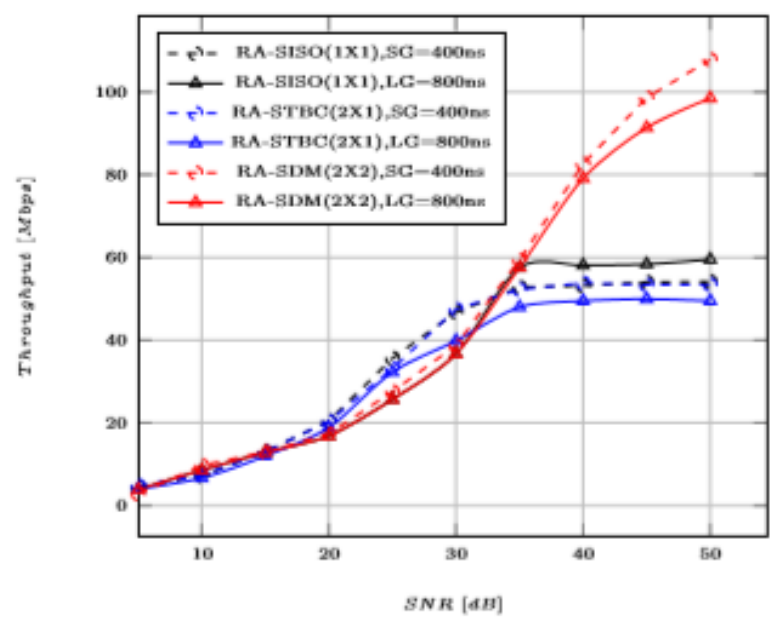

Fig 2. Throughput v/s SNR over TGn B with LOS 


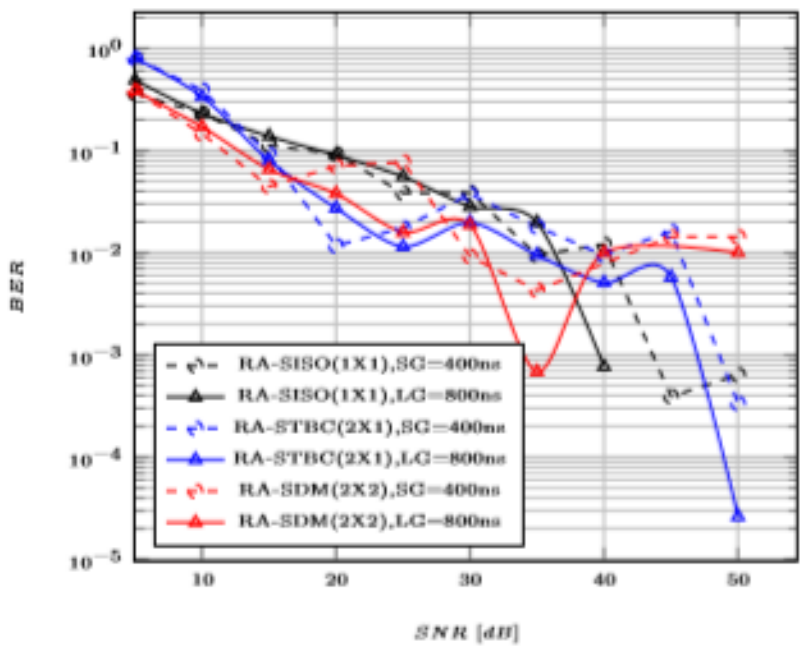

Fig 3. BER v/s SNR over TGn B channel with LOS

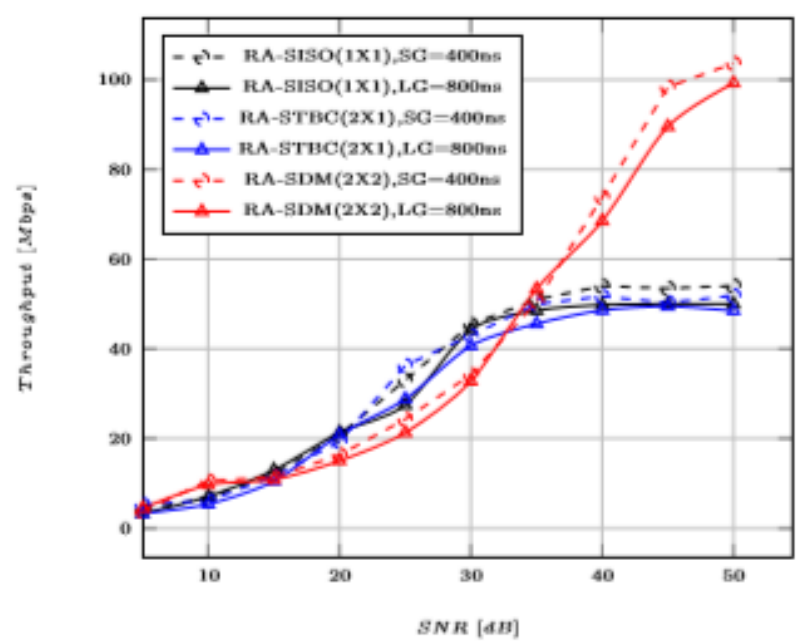

Fig 4. Throughput v/s SNR over TGn C channel with NLOS



Fig 5. BER v/s SNR over TGn C channel with NLOS

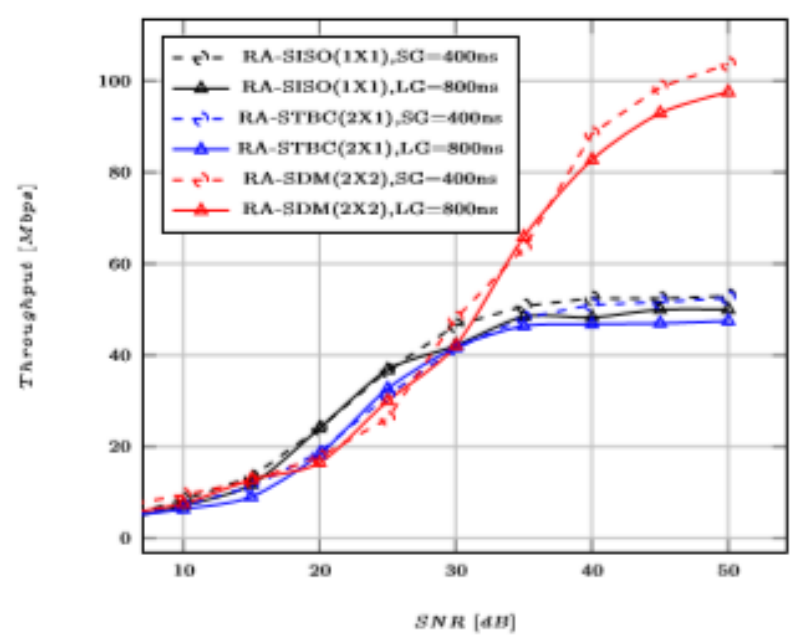

Fig 6. Throughput v/s SNR over TGn D channel with NLOS

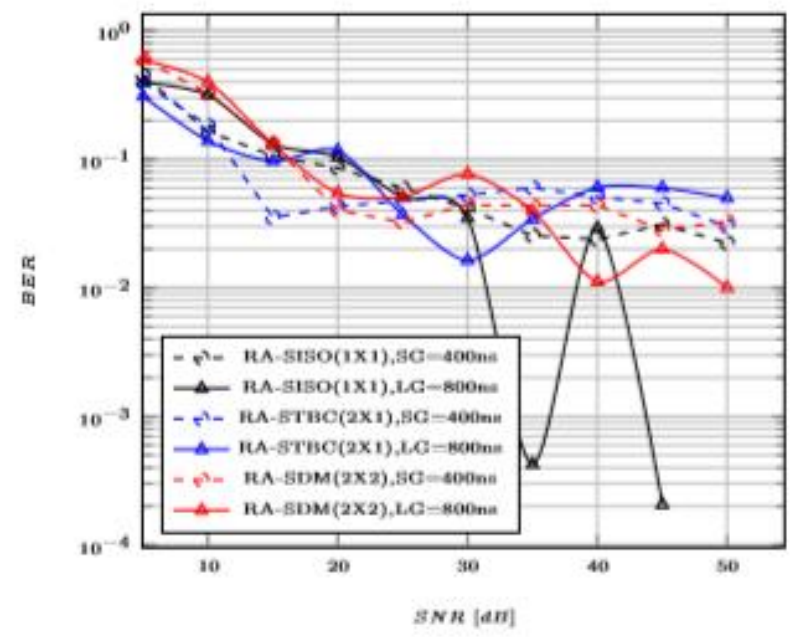

Figure 7. BER v/s SNR over TGn D channel with NLOS 
Optimal Combination of Transmission Parameters for Maximizing the Throughput of WLAN

Table V. Throughput performance of WLAN over TGn-B channel with LOS

\begin{tabular}{|c|c|c|c|c|c|c|c|}
\hline \multirow[t]{3}{*}{ SNR } & \multicolumn{6}{|c|}{ Throughput [Mbps] } & \multirow{3}{*}{$\begin{array}{c}\text { Optimum } \\
\text { transmit mode }\end{array}$} \\
\hline & \multicolumn{2}{|c|}{ RA-SISO } & \multicolumn{2}{|c|}{ RA-STBC } & \multicolumn{2}{|c|}{ RA-SDM } & \\
\hline & SG & LG & SG & LG & SG & LG & \\
\hline $5>$ SNR $<=10$ & 5.86 & 6.24 & 6.26 & 5.35 & 6.26 & 6.24 & $\begin{array}{c}\text { RA-STBC with } \\
\text { SG }\end{array}$ \\
\hline $10>$ SNR $<=20$ & 16.76 & 21.19 & 17.69 & 15.45 & 15.17 & 14.85 & $\begin{array}{l}\text { RA-SISO with } \\
\text { LG }\end{array}$ \\
\hline $20>$ SNR $<=30$ & 41.18 & 31.19 & 38.79 & 36.08 & 33.14 & 31.17 & $\begin{array}{l}\text { RA-SISO with } \\
\text { SG }\end{array}$ \\
\hline $30>$ SNR $<=40$ & 52.98 & 57.83 & 57.03 & 48.75 & 71.03 & 68.33 & $\begin{array}{c}\text { RA-SDM with } \\
\text { SG }\end{array}$ \\
\hline $40>$ SNR $<=50$ & 54.10 & 58.44 & 53.38 & 49.72 & 94.94 & 103.22 & $\begin{array}{c}\text { RA-SDM with } \\
\text { SG }\end{array}$ \\
\hline
\end{tabular}

Table VI. Throughput performance of WLAN over TGn-C channel with NLOS

\begin{tabular}{|c|c|c|c|c|c|c|c|}
\hline \multirow[t]{3}{*}{ SNR } & \multicolumn{6}{|c|}{ Throughput [Mbps] } & \multirow{3}{*}{$\begin{array}{c}\text { Optimum } \\
\text { transmit mode }\end{array}$} \\
\hline & \multicolumn{2}{|c|}{ RA-SISO } & \multicolumn{2}{|c|}{ RA-STBC } & \multicolumn{2}{|c|}{ RA-SDM } & \\
\hline & SG & LG & SG & LG & SG & LG & \\
\hline $5>$ SNR $<=10$ & 5.43 & 6.20 & 5.74 & 4.36 & 7.17 & 7.09 & $\begin{array}{c}\text { RA-SDM with } \\
\text { SG }\end{array}$ \\
\hline $10>$ SNR $<=20$ & 16.47 & 18.17 & 16.48 & 15.70 & 13.91 & 12.97 & $\begin{array}{l}\text { RA-SISO with } \\
\text { LG }\end{array}$ \\
\hline $20>$ SNR $<=30$ & 41.23 & 35.90 & 41.15 & 34.73 & 29.26 & 27.07 & $\begin{array}{c}\text { RA-SISO with } \\
\text { SG }\end{array}$ \\
\hline $30>\mathrm{SNR}<=40$ & 53.50 & 49.17 & 50.85 & 47.09 & 59.68 & 58.91 & $\begin{array}{l}\text { RA-SDM with } \\
\text { SG }\end{array}$ \\
\hline $40>$ SNR $<=50$ & 55.77 & 50 & 51.09 & 49.01 & 100.98 & 94.37 & $\begin{array}{c}\text { RA-SDM with } \\
\text { SG }\end{array}$ \\
\hline
\end{tabular}

Table VII. Throughput performance of WLAN over TGn-D channel with NLOS

\begin{tabular}{|c|c|c|c|c|c|c|c|}
\hline \multirow[t]{3}{*}{ SNR } & \multicolumn{6}{|c|}{ Throughput [Mbps] } & \multirow{3}{*}{$\begin{array}{c}\text { Optimum } \\
\text { transmit mode }\end{array}$} \\
\hline & \multicolumn{2}{|c|}{ RA-SISO } & \multicolumn{2}{|c|}{ RA-STBC } & \multicolumn{2}{|c|}{ RA-SDM } & \\
\hline & SG & LG & SG & LG & SG & LG & \\
\hline $5>$ SNR $<=10$ & 6.28 & 5.52 & 5.86 & 5.4 & 8.11 & 6.34 & $\begin{array}{c}\text { RA-SDM with } \\
\text { SG }\end{array}$ \\
\hline $10>$ SNR $<=20$ & 18.7 & 17.86 & 14.98 & 13.68 & 15.16 & 14.65 & $\begin{array}{c}\text { RA-SISO with } \\
\text { SG }\end{array}$ \\
\hline $20>\mathrm{SNR}<=30$ & 41.6 & 39.86 & 36.39 & 37.18 & 37.09 & 36.05 & $\begin{array}{c}\text { RA-SISO with } \\
\text { SG }\end{array}$ \\
\hline $30>\mathrm{SNR}<=40$ & 51.61 & 48.36 & 49.59 & 46.58 & 75.95 & 74.32 & $\begin{array}{c}\text { RA-SDM with } \\
\text { SG }\end{array}$ \\
\hline $40>$ SNR $<=50$ & 52.67 & 49.99 & 52.08 & 47.25 & 101.19 & 95.26 & $\begin{array}{c}\text { RA-SDM with } \\
\text { SG }\end{array}$ \\
\hline
\end{tabular}

\section{CONCLUSIONS}

This paper analyses the WLAN throughput and BER performance under different combinations of transmission parameters. The analysis shows that WLAN performance optimization is possible through optimal selection of different physical parameters (such as antenna configurations, guard interval between symbols, and modulation coding method) as a function of SNR. Various hybrid link adaptation proposals for WLAN [4] [19] [8] [6], succeefully improved the throughput of the system. However, our analysis shows that only the inclusion of multiple physical parameters in the link adaptation mechanism not necessarily gives better performance. By selecting the optimal combination of physical parameters or transmit mode adaptively as a function of SNR, WLAN performance can be optimized. Through this analysis, we have successfully obtained an optimal transmission mode lookup table offline (before transmission starts). This lookup table can reduce the computational burden on an adaptive controller that works on a per-packet basis.

\section{REFERENCES}

1. F. Peng, J. Zhang, W. E. Ryan, in Wireless Communications and Networking Conference, 2007. WCNC 2007. IEEE (IEEE, 2007), pp. 656-661.

2. S. H. Wong, H. Yang, S. Lu, V. Bharghavan, in Proceedings of the 12th annual international conference on Mobile computing and networking (ACM, 2006), pp. 146-157

3. I. W. Group, et al., IEEE Std. 802(2009)

4. L. Deek, E. Garcia-Villegas, E. Belding, S. J. Lee, K. Almeroth, in 2013 IEEE International Conference on Sensing, Communications and Networking (SECON) (IEEE, 2013), pp. 167-175 Analysis of link adaptation problem in WLAN 175.

5. I. Pefkianakis, Y.Hu, S. H. Wong, H. Yang, S. Lu, in Proceedings of the sixteenth annual international conference on Mobile computing and networking (ACM, 2010), pp. 257-268

6. L. Kriara, M.K. Marina, ACM SIGCOMM Computer Communication Review 45(2), 4 (2015)

7. D. Qiao, S. Choi, A. Jain, K.G. Shin, in Proceedings of the 9th annual international conference on Mobile computing and networking (ACM, 2003), pp. 161-175

8. T. Huang, S. Li, X. Lu, S. Gao, Wireless Communications and Mobile Computing 2019 (2019) 
9. Y. S. Cho, J. Kim, W.Y. Yang, C. G. Kang, MIMO-OFDM wireless communications with MATLAB (John Wiley \& Sons, 2010)

10. C. Oestges, B. Clerckx, MIMO wireless communications: from real-world propagation to space-time code design (Academic Press, 2010)

11. A. Goldsmith, Wireless communications (Cambridge university press, 2005)

12. J. G. Proakis, in Computer Engineering (McGraw-Hill, 2001)

13. R. van Nee, in Globalization of Mobile and Wireless Communications (Springer, 2011), pp. 103-118

14. E. Perahia, IEEE Communications Magazine 46(7) (2008)

15. V. Erceg, L. Schumacher, P. Kyritsi, et al., Garden Grove, California (2004)

16. M. K. Simon, M. S. Alouini, Digital communication over fading channels, vol. 95 (John Wiley \& Sons, 2005)

17. S. M. Alamouti, IEEE Journal on selected areas in communications 16(8), 1451 (1998)

18. D. Gesbert, M. Shafi, D. S. Shiu, P. J. Smith, A. Naguib, IEEE Journal on selected areas in Communications 21(3), 281 (2003)

19. T. Huang, H. Chen, L. Cui, Z. Zhang, International Journal of Sensor Networks 14(1), 9 (2013)

\section{AUTHORS PROFILE}

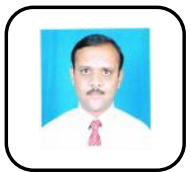

Pravinkumar Patil, received his master's degree in Electronics and Telecommunication Engineering from Shivaji University, Kolhapur (Maharashtra). Presently, he is associated with SCOEM, Satara (Shivaji University, Kolhapur) and handling the responsibility of the head of the Electronics and Telecommunication department. Previously, he was with SSPMCOE, Kankavli (University of Mumbai), where he worked as Assistant Professor in the Department of Electronics and Telecommunication. He is currently working towards Ph.D. degree at VTU, Belagavi. His research interest includes signal processing for communication applications, VLSI design and image processing Email-pspatil108@gmail.com Mobile-91-7972694007.

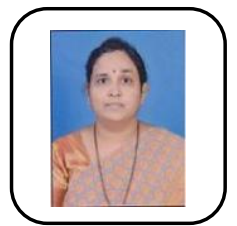

Dr. Meenakshi Patil, received post graduate degree in Electronics from Shivaji University and completed PhD Degree in 2011. She is currently Director Jain AGMIT Jamkhandi. She has worked as a session chair at the international Conference held at RCOE Mumbai. Involved in organizing international conferences, paper presentation contest, and worked as a Jury member for many technical programs. She has been involved in designing a few communication trainer kits. Currently, five research students are working on various fields in signal her supervision.

Email-meenakshirpatil@gmail.com

Mobile-91-9449806808.

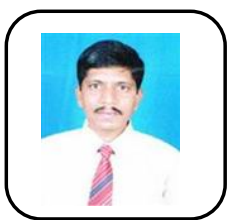

Santosh Itraj, is pursuing Ph.D. in Electronics Engineering at Shivaji University Kolhapur, Maharashtra, India. He received his master's degree in Electronics Engineering from Shivaji University Kolhapur. Presently he is working as an Associate Professor in the Department of Electronics and Telecommunication, SCOEM, Satara affiliated to Shivaji University Kolhapur. Previously he worked as an Associate professor at SSPMCOE Kankavali. His research interest is wireless communication, cognitive radio technology, signal processing, and embedded systems. Email-ssitraj@yahoo.com

Mobile-91-9422405192.

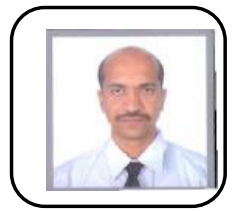

Dr. Uttam Bombale- received Ph.D. in Electronics Engineering from the Dhirubhai Ambani Institute of Information and Communication Technology, Gandhinagar, Gujarat, India. He received his master's degree in Electronics and Telecommunication in1994 from COE Pune. Presently he is working as Professor in the Department of Technology Shivaji University Kolhapur. His research interest is in the area of micro strip antennas, microwave technology, and wireless communication. He published papers in International journals and International conferences.

Email-uttam bombale@rediffmail.com Mobile-91-9049274380. 